\title{
Innovative approach to the difficult ventricular shunt using pleural access device for maintenance drainage: case report
}

\author{
Sharon R. Weeks, MD, ${ }^{1}$ Carolyn E. Gosztyla, MD, ${ }^{1}$ Laurence Davidson, MD, ${ }^{2}$ and \\ Howard I. Pryor II, MD³
}

Divisions of ${ }^{1}$ General Surgery, ${ }^{2}$ Neurosurgery, and ${ }^{3}$ Pediatric Surgery, Department of Surgery, Uniformed Services University of the Health Sciences, Walter Reed National Military Medical Center, Bethesda, Maryland

Patients with complex medical problems and multiple failed ventricular shunts require continued innovation for hydrocephalus management. The authors report the case of a 4-year-old boy with refractory hydrocephalus and secondary reduced ability to absorb CSF in both the pleural and peritoneal cavities following renal transplantation. A novel management approach was devised with split shunting to pleural and peritoneal targets as well as prophylactic pleural port placement to provide a method for minimally invasive thoracentesis should symptomatic pleural effusions develop. Fluid was successfully aspirated via the pleural port with relief of symptoms over a period of 16 months without complication. The authors demonstrate that a previously undescribed approach to distal shunting can prevent neurological sequelae of shunt failure and permit noninvasive maintenance drainage for patients in whom symptomatic pleural effusion is a recurrent complication.

https://thejns.org/doi/abs/10.3171/2019.10.PEDS19122

KEYWORDS hydrocephalus; shunt failure; ventriculoperitoneal shunt; ventriculopleural shunt

$\mathrm{C}$ ONGENITAL hydrocephalus and acquired hydrocephalus are common neurosurgical problems in children. CSF shunting is the cornerstone of therapy for communicating hydrocephalus, with multiple possible distal targets described. However, little innovation has been reported in this field, thus creating a therapeutic dilemma for a patient with multiple failures of described techniques. In this report, we detail an innovative approach to such a patient.

\section{Case Report}

History

Our male patient was born prematurely via spontaneous vaginal delivery at a gestational age of 24 weeks, 1 day. He had a left grade 4 and right grade 3 intraventricular hemorrhage and resultant posthemorrhagic hydrocephalus. A ventriculoatrial (VA) shunt was placed initially because his postnatal course was complicated by necrotizing enterocolitis requiring bowel resection and diverting ostomy.
Over the years, the patient experienced multiple distal VA shunt malfunctions secondary to thrombosis, thought to be due to heterozygosity for Factor V Leiden. His initial VA shunt was eventually converted to a ventriculoperitoneal (VP) shunt, which failed due to extensive intraabdominal adhesions. A ventriculopleural (VPI) shunt was then placed and well tolerated. After renal transplantation at the age of 4 years for dysplastic kidneys, however, the patient repeatedly presented with symptomatic pleural effusions requiring therapeutic thoracenteses, as frequently as every 48 hours. It was in this condition that he transferred to our facility for further management.

At our institution, the patient underwent multiple attempts at surgical revision, first to the peritoneal and then the atrial distal targets, each leading to distal shunt failure within the 1st postoperative week. His shunt was initially revised to a VP shunt, but the patient developed shunt failure on postoperative day (POD) 6. Rescue of the VP shunt was attempted with lysis of abdominal adhesions but this approach was unsuccessful, and the patient developed

ABBREVIATIONS POD = postoperative day; VA = ventriculoatrial; VP = ventriculoperitoneal; VPI = ventriculopleural.

SUBMITTED June 6, 2019. ACCEPTED October 15, 2019.

INCLUDE WHEN CITING Published online January 3, 2020; DOI: 10.3171/2019.10.PEDS19122. 

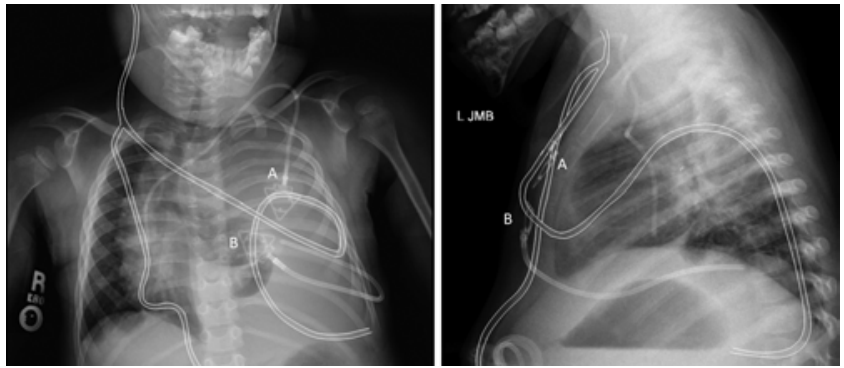

FIG. 1. Chest radiographs showing configuration of devices from posteroanterior (left) and lateral (right) views. The ventricular shunt is outlined in white and splits at the Y-connector into peritoneal and pleural distal targets. Also seen are the implantable port to the left subclavian for durable vascular access $(A)$ and implantable port to the left pleural space placed prophylactically for maintenance drainage of CSF accumulation (B).

shunt failure on POD 20 from the first surgery and POD 14 from the second; his shunt was revised to a VA shunt. Failure of the VA shunt occurred due to extensive thrombus formation in the distal catheter on POD 22 from the first surgery, POD 16 from the second, and POD 2 from the third, despite the use of prophylactically dosed enoxaparin. Use of the gallbladder as the distal target was ruled out, as the risk for infectious complications was considered unacceptable in this immunosuppressed transplant patient. Furthermore, given the small size and deep location of his gallbladder in the setting of extensive abdominal adhesions, the gallbladder was not readily accessible. He was initially not deemed a candidate for endoscopic third ventriculostomy and choroid plexus cauterization after referrals to multiple quaternary care centers. Given the lack of durability of traditional approaches, a novel approach for management was devised with splitting of the distal shunt into pleural and peritoneal catheters and placement of a separate pleural port to drain fluid accumulation from the pleural space.

\section{Operative Approach}

Intraoperatively, the shunt was connected to a Y-connector located distal to the programmable valve. This Yconnector was then connected to the previously placed peritoneal catheter (which, when previously ligated, had been left in place for possible future use) and to a pleural catheter that was tunneled in the subcutaneous tissue across the anterior left chest. Using video-assisted thoracoscopic surgery techniques, the left pleural space was accessed via a 4-mm incision in the sixth intercostal space at the anterior axillary line. The tunneled catheter was placed in the left pleural cavity under direct visualization through a second 4-mm incision. Through a third 4-mm incision at the posteroinferior aspect of the eighth intercostal space, the distal tip of an 8-Fr implantable subcutaneous port was placed in the pleural space, and the reservoir was secured to the anterior chest wall (Fig. 1). The patient had an uncomplicated postoperative recovery.

\section{Postoperative Course}

Four months postoperatively, the patient presented to
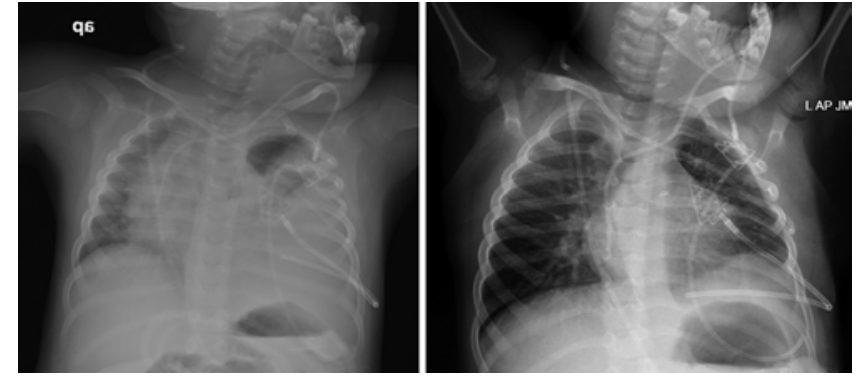

FIG. 2. Chest radiographs obtained 4 months postoperatively when the patient presented with dyspnea. Radiographs demonstrated pleural effusion (left) and resolution following aspiration of fluid via the pleural port (right).

the clinic with respiratory symptoms and radiographs showing accumulation of pleural fluid. Fluid was successfully aspirated in the clinic via the pleural port with relief of symptoms (Fig. 2). He required drainage of pleural fluid a median of every 5 days (IQR 5-7 days), with a median drainage of $582 \mathrm{ml}$ per week (IQR 451-710 ml). Ongoing drainage requirements were persistent, but at 16 months postoperatively, the patient had no further instances of shunt failure. These drainage procedures are performed initially in the outpatient clinic in a minimally invasive fashion via sterile access of the subcutaneous pleural port. Eventually, the parents assumed care of his pleural effusions by accessing the port and performing pleural drainage at home. Addition of acetazolamide to his medication regimen has further decreased output.

\section{Discussion}

Pediatric surgeons are often consulted to assist in the placement and management of ventricular shunts in complicated patients. These patients typically do well with rare distal complications and good outcomes. However, advances in care of the neonatal patient with multiple comorbidities have led to the need for management of increasingly complex patients with long-standing shunts. In this report, we detail a novel approach in a 4-year-old boy with numerous VA, VPl, and VP shunt malfunctions due to hypercoagulability and insufficient CSF absorption capacity in pleural and peritoneal cavities. Interestingly, this issue directly followed a renal transplant and the start of immunosuppressive medication.

The likely cause of failure for the peritoneal target was extensive intraabdominal adhesions preventing adequate surface area for CSF absorption, even after lysis of adhesions, as the patient was free of constipation or infections. ${ }^{3}$ Central venous system targets were plagued by thrombosis in this patient with a hypercoagulable condition, despite the use of prophylactically dosed enoxaparin. The use of therapeutic anticoagulation was considered, although this indication has not been studied. ${ }^{3}$ The risks of full anticoagulation were thought to outweigh the benefits in this developmentally delayed child at high risk of falls that could result in catastrophic bleeding. Finally, the pleural space was an ineffective target as well, which is not uncommon for children younger than 3 to 7 years due to small pleural 
surface area, although there is conflicting literature on this point. ${ }^{3,5-7}$ Nonetheless, the patient did very well with a VPl shunt until he received a renal transplant. Ventriculosinus shunts have also been described, although the published literature is limited in follow-up and the procedure is not commonly done at most centers. ${ }^{10}$ These shunts from cerebral ventricles to the cerebral venous sinuses, in theory, mimic physiological conditions, but such an approach may have been problematic in our patient with proven risk for thrombosis. Finally, ventriculocholecystic shunts are not common, with fewer than 100 cases reported in the literature. ${ }^{4}$ Although there is a case series of 25 patients who experienced good outcomes, ${ }^{13}$ ventriculitis from bile reflux has been associated with mortality. ${ }^{1}$ Due to our patient's immunocompromised state and unfavorable anatomy, his gallbladder was not thought to be a feasible distal shunt target.

Ventricular shunting has been the mainstay of surgical therapy since the $1950 \mathrm{~s}$ and is the primary option available for children with communicating hydrocephalus. ${ }^{11}$ The distal catheter is inserted into a cavity to serve as a receptacle for CSF and ultimately permits absorption into the bloodstream. Our patient had attempts at all conventional targets with subsequent failure and required 4 shunt revisions in 1 month. A case report by Ratliff et al. ${ }^{9}$ described the use of a ventriculo-bipleural shunt in a 4-yearold boy in whom VP, VA, and VPl shunts failed, a similar clinical scenario to that of our patient. As in our case, they utilized a Y-connector distal to the shunt valve to drain CSF to 2 targets, both pleural spaces in their case. In the 5 -year follow-up, the shunt has remained functional without signs of dyspnea.

In our patient, we had concern for the possibility of simultaneous bilateral pleural effusions resulting in respiratory failure and therefore devised a method in which CSF was drained into both a pleural space and the peritoneal cavity via a Y-connector located distal to the programmable valve. As this splitting is distal to the valve, drainage may flow to the peritoneal cavity, pleural cavity, or both. Importantly, in shared clinical decision-making with the family, we found that anticipating possible failure and having a management plan in place prior to failure in this complex patient was critical. With no targets remaining after this dual target approach, we innovated a "safety net" mechanism in the event of pleural effusion-an implantable catheter in the pleural space to provide a minimally invasive option for thoracentesis. Additionally, as part of a multimodal approach, the patient was also placed on a regimen of acetazolamide to decrease CSF production, which has been shown in case reports to help the success of VPl shunts in infants with failure at other targets. ${ }^{6}$

The innovative addition of a minimally invasive method to drain recurrent pleural effusions, while not a definitive treatment, provided a year-long therapeutic bridge without invasive intervention as we awaited adhesion remodeling, pleural surface area growth, and further evaluation for choroid plexus cauterization with endoscopic third ventriculostomy. ${ }^{2,8,12}$ There are several advantages to this minimally invasive technique in comparison with repeated thoracentesis. First, while thoracentesis requires advanced training and privileges, comfort and training with port access are more common among providers. It can also be performed by qualified nurses, who commonly access ports. Port access and drainage can even be taught to family members, depending on comfort and experience, as we were able to do. Port access is tolerated better by the patient and is associated with a lower risk of complications than with a more invasive thoracentesis procedure. These advantages improve patient safety as well as the quality of life for complex patients and their families.

For this unique clinical scenario, we demonstrated that a previously undescribed approach to distal shunting can prevent neurological sequelae of shunt failure and permit maintenance drainage for patients with a difficult shunt history in whom symptomatic pleural effusion is a recurrent complication.

\section{References}

1. Bernstein RA, Hsueh W: Ventriculocholecystic shunt. A mortality report. Surg Neurol 23:31-37, 1985

2. Chamiraju P, Bhatia S, Sandberg DI, Ragheb J: Endoscopic third ventriculostomy and choroid plexus cauterization in posthemorrhagic hydrocephalus of prematurity. J Neurosurg Pediatr 13:433-439, 2014

3. Hanak BW, Bonow RH, Harris CA, Browd SR: Cerebrospinal fluid shunting complications in children. Pediatr Neurosurg 52:381-400, 2017

4. Hasslacher-Arellano JF, Arellano-Aguilar G, FunesRodríguez JF, López-Forcén S, Torres-Zapiain F, Domínguez-Carrillo LG: Ventriculo-gallbladder shunt: an alternative for the treatment of hydrocephalus. Cirugía y Cir (English Ed) 84:225-229, 2016

5. Hoffman HJ, Hendrick EB, Humphreys RP: Experience with ventriculo-pleural shunts. Childs Brain 10:404-413, 1983

6. Jones RF, Currie BG, Kwok BC: Ventriculopleural shunts for hydrocephalus: a useful alternative. Neurosurgery 23:753755,1988

7. Kalra RRS, Kestle J: Treatment of hydrocephalus with shunts, in Albright AL, Pollack IF, Adelson PD (eds): Principles and Practice of Pediatric Neurosurgery, ed 3. New York: Thieme Medical Publishers, 2015, pp 100-118

8. Kulkarni AV, Riva-Cambrin J, Browd SR, Drake JM, Holubkov R, Kestle JRW, et al: Endoscopic third ventriculostomy and choroid plexus cauterization in infants with hydrocephalus: a retrospective Hydrocephalus Clinical Research Network study. J Neurosurg Pediatr 14:224-229, 2014

9. Ratliff M, Unterberg A, Bächli H: Ventriculo-bipleural shunt as last resort in a 4-year-old child in whom a VP and VA shunt failed. J Neurosurg Pediatr 17:285-288, 2016

10. Toma AK, Tarnaris A, Kitchen ND, Watkins LD: Ventriculosinus shunt. Neurosurg Rev 33:147-153, 2010

11. von Allmen D: Neurosurgery for the pediatric surgeon, in Ziegler M, Azizkhan R, von Allmen D, Weber T (eds): Operative Pediatric Surgery, ed 2. New York: McGrawHill, 2014

12. Waqar M, Ellenbogen JR, Mallucci C: Endoscopic third ventriculostomy for shunt malfunction in children: a review. J Clin Neurosci 51:6-11, 2018

13. West CG: Ventriculovesical shunt. Technical note. J Neurosurg 53:858-860, 1980

\section{Disclaimer}

The opinions or assertions contained herein are the private ones of the authors and are not to be construed as official or reflecting the views of the Department of Defense, Department of the 
Army, Department of the Navy, the Uniformed Services University of the Health Sciences, or any other agency of the U.S. Government.

\section{Disclosures}

The authors report no conflict of interest concerning the materials or methods used in this study or the findings specified in this paper.

\section{Author Contributions}

Conception and design: Pryor, Weeks, Davidson. Acquisition of data: Pryor, Weeks, Davidson. Analysis and interpretation of data: all authors. Drafting the article: Weeks, Gosztyla. Critically revis- ing the article: all authors. Reviewed submitted version of manuscript: all authors. Study supervision: Pryor.

\section{Correspondence}

Howard I. Pryor II: Uniformed Services University of the Health Sciences, Walter Reed National Military Medical Center, Bethesda, MD. howard.i.pryor.mil@mail.mil. 